\title{
Trial protocol OPPTIMUM- Does progesterone prophylaxis for the prevention of preterm labour improve outcome?
}

\author{
Jane E Norman ${ }^{1 *}$, Andrew Shennan ${ }^{2}$, Phillip Bennett ${ }^{3}$, Steven Thornton ${ }^{4}$, Stephen Robson ${ }^{5}$, Neil Marlow ${ }^{6}$, \\ John Norrie ${ }^{7}$, Stavros Petrou ${ }^{8}$, Neil Sebire ${ }^{9}$, Tina Lavender ${ }^{10}$ and Sonia Whyte ${ }^{11}$
}

\begin{abstract}
Background: Preterm birth is a global problem, with a prevalence of 8 to 12\% depending on location.. Several large trials and systematic reviews have shown progestogens to be effective in preventing or delaying preterm birth in selected high risk women with a singleton pregnancy (including those with a short cervix or previous preterm birth). Although an improvement in short term neonatal outcomes has been shown in some trials these have not consistently been confirmed in meta-analyses. Additionally data on longer term outcomes is limited to a single trial where no difference in outcomes was demonstrated at four years of age of the child, despite those in the "progesterone" group having a lower incidence of preterm birth.

Methods/Design: The OPPTIMUM study is a double blind randomized placebo controlled trial to determine whether progesterone prophylaxis to prevent preterm birth has long term neonatal or infant benefit. Specifically it will study whether, in women with singleton pregnancy and at high risk of preterm labour, prophylactic vaginal natural progesterone, $200 \mathrm{mg}$ daily from 22 - 34 weeks gestation, compared to placebo, improves obstetric outcome by lengthening pregnancy thus reducing the incidence of preterm delivery (before 34 weeks), improves neonatal outcome by reducing a composite of death and major morbidity, and leads to improved childhood cognitive and neurosensory outcomes at two years of age. Recruitment began in 2009 and is scheduled to close in Spring 2013. As of May 2012, over 800 women had been randomized in 60 sites.
\end{abstract}

Discussion: OPPTIMUM will provide further evidence on the effectiveness of vaginal progesterone for prevention of preterm birth and improvement of neonatal outcomes in selected groups of women with singleton pregnancy at high risk of preterm birth. Additionally it will determine whether any reduction in the incidence of preterm birth is accompanied by improved childhood outcome.

Trial registration: ISRCTN14568373

\section{Background, including rationale and any previous systematic review(s)}

Preterm birth is a global problem, with a prevalence of 8 to $12 \%$ depending on location [1]. Around $75 \%$ of preterm birth follows spontaneous preterm labour, sometimes preceded by preterm premature membrane rupture [2]. Babies born preterm are at increased risk of a variety of adverse short term (neonatal) and long term complications, including neurodevelopmental disability.

\footnotetext{
* Correspondence: jane.norman@ed.ac.uk

'University of Edinburgh MRC Centre for Reproductive Health, The Queen's Medical Research Institute, 47 Little France Crescent, Edinburgh EH16 4TY, UK Full list of author information is available at the end of the article
}

Women with a previous preterm birth (especially those women who delivered before 34 weeks following spontaneous preterm labour), women with a short cervix in early pregnancy [3], and women with a previous cone biopsy or laser loop excision to the cervix [4] are at all at increased risk of spontaneous preterm birth.

Several large trials and meta-analyses have shown progesterone to be effective in preventing or delaying preterm birth in selected high risk women (short cervix or previous preterm birth) with singleton pregnancy [5-11]. There is data that both intramuscular 17 hydroxyprogesterone caproate and vaginal progesterone are effective in preventing preterm birth. OPPTIMUM likewise is 
investigating the efficacy of progesterone in women at elevated risk of preterm birth, but will crucially address longer term childhood developmental outcomes.

Although an improvement in short term neonatal outcomes has been shown in some trials [6-8] these have not consistently been confirmed in meta-analyses $[9,10]$. Data on longer term outcomes in singletons is limited to follow up of babies of women in the Meis trial, where $80 \%$ of babies were assessed by questionnaire at a mean age of four years. No differences in childhood outcomes were demonstrated, despite the progesterone group having a lower incidence of preterm birth [12].

The mechanisms of action of progesterone are somewhat uncertain although a direct inhibitory effect on the processes of parturition seem likely [13]. Additionally progesterone could exert anti-inflammatory properties [13] and/or direct CNS protective effects [14], which could help to reduce the risk or severity of long term neonatal problems.

If the prevention of preterm birth is accompanied by a reduction in the complications of prematurity, then in utero progesterone should be predicted to have long term beneficial effects. Harmful long term effects are however also possible. Although direct teratogenic effects of progesterone are unlikely, there could be adverse effects of keeping the fetus in utero in a compromised intrauterine environment where infection or inflammation is present. Caution is therefore warranted before progesterone use becomes widespread, particularly since drugs (including antibiotics and estrogens) thought to be beneficial in women at risk of preterm birth have now been demonstrated to have long term adverse effects [15-17].

The OPPTIMUM study is designed to determine whether progesterone prophylaxis to prevent preterm birth has long term neonatal or childhood benefit. Specifically it will study whether, in women at elevated risk of preterm labour, prophylactic vaginal natural progesterone, $200 \mathrm{mg}$ (compared to placebo), daily from 22 24 weeks up to 34 weeks gestation, improves obstetric outcome by lengthening pregnancy thus reducing the incidence of preterm delivery (before 34 weeks), improves neonatal outcome by reducing a composite of death and major morbidity and leads to improved childhood cognitive and neurosensory outcomes at two years of age.

The OPPTIMUM study began recruiting in January 2009. Since then, one large, and several other smaller studies have reported the effect of progesterone, either as vaginal progesterone or as intramuscular 17 hydroxyprogesteronecaproate. None fully addresses the crucial question regarding long term outcome (childhood development at 2 years). Additionally, we note that the US Food and Drug Administration (FDA) has indicated that long term childhood outcome data are required to determine the clinical benefits and risks of 17 hydroxyprogesteronecaproate for the prevention of preterm birth in women with a previous preterm birth. ${ }^{\text {a }}$ An FDA panel also recently ruled that data supplied on vaginal progesterone gel "do not [yet] support the efficacy of progesterone gel compared with placebo in reducing the risk of preterm births before 33 completed weeks of gestation among women with a short cervical length". 'We believe that OPPTIMUM will address these important evidence gaps about the efficacy of vaginal progesterone and any childhood effects of progestogens in general.

\section{Methods}

$\operatorname{Aim}(\mathbf{s})$

The aim of the OPPTIMUM study is to determine whether, in women at high risk of preterm labour, prophylactic vaginal natural progesterone, $200 \mathrm{mg}$ daily from $22-34$ weeks gestation, compared to placebo:

- Improves obstetric outcome by reducing the incidence of preterm delivery (before 34 weeks gestation).

- Improves neonatal outcome by reducing a composite of death and major morbidity.

- Leads to improved childhood cognitive and neurosensory outcomes at two years.

\section{Centre(s)}

More than 60 hospitals, principally in the UK.

\section{Design}

Double blind randomized placebo controlled trial. The study is in two phases, a screening phase and a treatment phase.

\section{Inclusion and exclusion criteria \\ Screening phase inclusion criteria}

High risk for preterm birth (as indicated by AT LEAST

ONE of the criteria i-iv) and ALL of the criteria v-vii:

i. History of previous PTB/second trimester loss ( $\geq 16$ weeks or $\leq 37$ weeks gestation)

ii. Previous preterm premature rupture of the fetal membranes ( $\leq 37$ weeks gestation)

iii. Short cervical length $(\leq 25 \mathrm{~mm})$ on ultrasound at 18-0 - 24 + 0 weeks gestation

iv. Any cervical procedure to treat abnormal smears i.e. large loop excision, laser conisation, cold knife conisation or radical diathermy

v. Gestation established by scan at $\leq 16$ weeks to ensure that the estimated date of delivery is accurate or the consultant must be confident that the gestation dates are accurate.

vi. Signed Consent form vii.16 years of age or older. 


\section{Treatment phase inclusion criteria}

- All women fulfilling the inclusion criteria for the screening phase and who also have a positive screening (fFN) test at $22+0$ weeks, will be eligible for the main (treatment) phase of the study - these women are subsequently referred to as the "fFN positive" group.

- Additionally, in September 2010, those who have a previous spontaneous labour resulting in a preterm birth $\leq 34$ weeks gestation (delivery by any mode) or a short cervix in index pregnancy, defined as cervical length $\leq 25 \mathrm{~mm}$ at $18-0-24+0$ weeks gestation also became eligible for randomisation even with a negative fFN test. These women are subsequently referred to as the "fFN negative" group

\section{Exclusion criteria}

- Known significant congenital structural or chromosomal fetal anomaly

- Known sensitivity or listed contraindication to progesterone (known allergy or hypersensitivity to progesterone, severe hepatic dysfunction, undiagnosed vaginal bleeding, mammary or genital tract carcinoma, thrombophlebitis, thromboembolic disorders, cerebral haemorrhage, porphyria) or intolerance to progesterone or excipient (including peanut allergy prior to February 2011 given that peanut oil was the excipient in doses issued to participants until November 2010)

- Suspected or proven rupture of the fetal membranes at the time of recruitment

- Multiple pregnancy

- Prescription or ingestion of medications known to interact with progesterone (e.g. Bromocriptine, Rifamycin, Ketoconazole or Ciclosporin)

- Women currently prescribed progesterone or who have taken progesterone beyond 18 weeks gestation.

Intervention(s) Progesterone (Utrogestan) $200 \mathrm{mg}$ soft capsules or placebo will to be inserted once daily vaginally at bedtime from $22^{+0}-24^{+0}$ up to $34^{+0}$ weeks gestation.

\section{Randomisation}

Randomisation will be carried out online via the web portal or via telephone to the central randomisation facility based at the Robertson Centre for Biostatistics, at the Glasgow Clinical Trials Unit, University of Glasgow.

\section{Concealment of allocation}

Concealment of allocation will be achieved by randomising participants to active or placebo capsules. Placebo capsules will appear identical to active treatment. The outcomes will be measured blind to the allocation.

\section{Primary and any secondary endpoint(s)}

Primary endpoints:

- Obstetric: delivery <34 completed weeks of gestation (Yes/No), $(<34+0$ weeks: outcome of the treatment phase)

- Neonatal: a composite of death or two markers of neonatal morbidity - bronchopulmonary dysplasia in children born at $<32$ weeks of gestation and brain injury on cerebral ultrasound.

- Childhood: The Bayley III cognitive scale standardised score at two years of chronological age (with an aim to test between 22 months to 26 months - as this is age-standardised all assessments will be valid)

Secondary endpoints:

- Gestation at delivery

- Fetal or neonatal/infant death after trial entry up to 2 years of age [18].

- Incidence of the individual components of the primary neonatal outcome

- Incidence of other major neonatal complications:

- Level of care days, which includes: days of respiratory support, (Either mechanical ventilation or CPAP) and days of oxygen therapy.

- Surfactant administration

- Necrotising enterocolitis, (medical or surgical treatment of confirmed cases)

- Number of discrete episodes of bloodstream or CNS infection (positive blood [19] or CSF culture)

- Daily level of care [20].

- Composite outcome of death or moderate/severe neurodevelopmental impairment at two years of age, defined as per national recommendations [21].

- Individual components of the disability definition and non-neurological disability as defined [21].

- Strengths and difficulties questionnaire (http://www. sdqinfo.com/)

- Score on the PARCA-r (parent assessment of child abilities revised).

- Women's perceptions of their treatment.

- Maternal and child adverse events (e.g. operative delivery).

Side-effects reporting and quantification (e.g, WHO scale) Participants are instructed to contact their Investigator at any time after consenting to join the trial if any symptoms or side effects develop. In the case of any events the Investigator should initiate the appropriate treatment 
according to their medical judgment. All adverse events (AEs) and Serious Adverse Events (SAE) that occur after being randomized to treatment must be recorded in detail in the patient notes. SAEs occurring in mother or baby from the time a participant is randomised until 30 days after stopping taking study treatment/placebo or until 28 days after delivery (whichever is the later) should be reported to the co-sponsors using the trial documentation. The standard definition of a serious adverse event will be used [22]. This is any event that: results in death; is life threatening (i.e. the subject was at risk of death at the time of the event; it does not refer to an event which hypothetically might have caused death if it were more severe); requires hospitalisation or prolongation of existing hospitalisation; results in persistent or significant disability or incapacity; is a congenital anomaly or birth defect. Hospitalisations for treatment planned prior to randomisation and hospitalisation for elective treatment of a pre-existing condition will not be considered as an SAE, nor, for the purposes of this study, will the following events: miscarriage, preterm labour / suspected preterm labour, premature rupture of membranes (PROM) / suspected PROM, preterm delivery, preterm delivery in maternal interest, preterm delivery in fetal interest, hospitalisation for pregnancy induced hypertension, hospitalisation for "maternal discomfort", hospitalisation for "rest", hospitalisation for "observation" or "monitoring" for which the women are admitted for a period of less than 12 hours; normal" childhood illnesses (including infectious diseases and minor injuries) and complications arising from prematurity.

\section{Statistical analysis plan, including sample size and power calculations}

At the commencement of the study in 2008, we planned to recruit 750 women in the "fFN positive" group. We estimated that such a sample size would provide $80-95 \%$ power at the $5 \%$ level of significance for the primary obstetric outcome, $80 \%$ power for the neonatal outcome and 93\% power for the childhood outcome (although further dilution of the latter was anticipated after incorporation of the deaths in a two stage statistical model - see below).

In 2010, the study was expanded to allow randomisation of women in the "fFN negative" group after analysis of preliminary (blinded) data in July 2010, together with the result of an HTA funded systematic review on screening for preterm birth [23] showed that our initial selection strategy erroneously missed women at medium to high risk of preterm birth. This change was endorsed by the Trial Steering committee, the MHRA, the ethics committee, and by the funder. The inclusion of women at a lower risk of the primary outcomes necessitated an increase in the sample size. The minimum new sample size is anticipated to be in the order of 1125 (375 fFN positive and 750 fFN negative women), but the proportions of each will dictate the exact sample size, as it is anticipated that at least $2 \mathrm{fFN}$ negative women are required to achieve the event rate predicted in $1 \mathrm{fFN}$ positive woman. Workings for the sample size calculation for the fFN positive group alone, and for the revised combined group of fFN positive and fFN negative women are shown in the Appendix.

\section{Type of analysis (e.g. intention to treat) and statistical tests}

All primary efficacy analyses are carried out on the intention to treat population (ITT). Safety analyses are carried out on the safety population (those who initiated on what study medication they received). Primary analyses are repeated in an exploratory manner on the per protocol population (including [i] compliance within an acceptable range and [ii] fulfilment of inclusion and exclusion criteria).

\section{Obstetric outcome}

The primary obstetric outcome is delivery or fetal death before 34 completed weeks of gestation based on ultrasound (based on the projected date of delivery estimated from scan in the first trimester). The following null hypothesis will be tested: 'There is no difference in the incidence of delivery or fetal death before 34 completed weeks of gestation between the group treated with $200 \mathrm{mg} /$ day progesterone and the group treated with placebo from week 22-24 to week 34 of gestation or earlier delivery'.

The outcome is compared between the treatment groups using a logistic regression model including treatment and previous pregnancy of at least 14 weeks. The hypothesis will be tested with a likelihood ratio test.

\section{Neonatal outcome}

The primary neonatal outcome is a binary outcome indicating whether one of the following has occurred:

- Death

- Brain injury (defined as any intraventricular haemorrhage (IVH) (excludes subependymal haemorrhages), parenchymal cystic or haemorrhagic lesion or persistent ventriculomegaly (VI $>97$ th percentile)

- Severe chronic lung disease (defined as need for $\geq 30 \%$ oxygen and/or positive pressure (positive pressure ventilation or nasal continuous positive airway pressure) at 36 weeks post menstrual age or discharge, which ever comes first).

The following null hypothesis will be tested: 'There is no difference in the combined incidence of neonatal 
death, brain injury or severe chronic lung disease between the group treated with $200 \mathrm{mg} /$ day progesterone and the group treated with placebo from week 22-24 to week 34 or earlier delivery' This outcome is also compared between the treatment groups using a logistic regression model including treatment and previous pregnancy of at least 14 weeks. The hypothesis will be tested with a likelihood ratio test.

\section{Childhood outcome}

The primary childhood outcome is the Bayley III score, a continuous measure. This outcome will, by definition, not be available on babies who have died. Thus deaths need to be incorporated into the analysis, since the number of deaths may be sufficiently large as not to be negligible, and/or there may be a difference in the number of deaths between the two randomised groups. We will therefore use a two-stage statistical model that jointly models the treatment effect in both deaths and survivors [24], with deaths modelled using a binomial test and survivors modelled using a generalised linear model. The two parts are then combined to form the appropriate test statistic. Secondary analyses that adjust the estimated treatment effect for covariates felt to be of importance will be used as appropriate. Note that we will not be adjusting for gestational age in our analysis of childhood outcomes. The hypothesised mechanism of action of progesterone is to increase gestational age by reducing the proportion of women giving birth prematurely. To adjust for a post randomisation covariate (gestational age) which is a direct measure of the treatment effect, in a model that is estimating the consequence of that treatment effect (in terms of developmental outcome) is not statistically sound.

A fully detailed Statistical Analysis Plan will be prepared prior to unblinding.

\section{Planned subgroup analyses}

In order to determine whether a reduced or improved response to progesterone can be predicted, subgroups of the ITT population will be formed according to the following factors:

- reason for risk of preterm delivery (spontaneous preterm birth yes / no and any preterm birth yes / no)

- previous pregnancy of at least 14 weeks (yes / no)

- cervical length at 18-24 weeks gestation ( $\leq 25 \mathrm{~mm} />25 \mathrm{~mm}$ and $\leq 15 \mathrm{~mm} />15 \mathrm{~mm}$ )

- chorioamnionitis diagnosed on pathology (yes / no)

Ethical issues, including: Ethics committee approval The study has been approved by the Scotland (A) Research Ethics Committee, reference number 08/MRE00/6.

\section{Interim analyses and stopping rules}

Interim unblinded analyses will be performed on safety, efficacy and possibly futility criteria for the purposes of review by the independent Data Monitoring Committee (iDMC) only. Masking will be maintained and analysis of obstetric and neonatal outcomes will be deferred until conclusion of the two-year childhood assessments. Thus no interim analyses will be revealed to investigators, participants, or anyone other than the DMC members (and statistical advisors) until completion of the two-year childhood assessments.

\section{Committee oversights}

There is an independent Trial Steering Committee and independent Data Monitoring Committee.

\section{Indemnities}

The sponsor has clinical trial insurance which covers this study. This insurance includes indemnification for the TSC and DMC. There is no "no fault" indemnity.

\section{Publication plan}

The results of the trial will be published in peer reviewed journals. The results of the obstetric and neonatal outcomes will not be published until data collection for the childhood follow up part of the study is complete.

\section{Funding}

The study is funded by the MRC/EME -funder reference G0700452, grant No: 84982

\section{Start date}

Recruitment began in January 2009, as of May 2012, over 800 women had been randomized

\section{End of data collection}

Estimated October 2015

\section{Reporting date}

Estimated mid 2016

\section{Trial registration}

ISRCTN14568373

\section{Discussion}

Despite the many publications on the use of progesterone for the prevention of preterm birth, we believe that that further data, particularly on the long term effect for the baby, is needed before the use of progesterone can be routinely recommended in pregnant women at high risk of preterm birth. Many authorities, including the FDA $^{\mathrm{a}}$ and the Royal College of Obstetricians in the $\mathrm{UK}^{\mathrm{c}}$ agree. The Society of Obstetricians and Gynecologists of 
Canada (SOGC) [25] suggests that women at risk of should be encouraged to enroll in trials of progesterone, although the SOGC also gives advice about the dose and formulation of progestogens for women who have opted to take them. Other authorities endorse the use of progesterone based on current literature. For example the American College of Obstetricians and Gynecologists, in guidelines published in 2008 and reaffirmed in 2011 [26] is more positive about the use of progesterone, saying that "progesterone supplementation for the prevention of recurrent preterm birth should be offered to women with a singleton pregnancy and a prior spontaneous preterm [before 37 weeks] birth" and that "progesterone supplementation for asymptomatic women with a very short cervical length (less than $15 \mathrm{~mm}$ ) may be considered". In 2012, the Society of Maternal and Fetal Medicine Publications committee suggests that women with singleton pregnancies with prior preterm birth be given 17 alpha hydroxyprogesterone, and that those with a short cervix $\leq 20 \mathrm{~mm}$ be given vaginal progesterone (although they indicate that routine screening is "an object of debate") [13].

The OPPTIMUM study will provide further evidence on the effectiveness of vaginal progesterone for the prevention of preterm delivery in selected high risk women. Crucially, it will also determine whether prevention of preterm birth by progesterone prophylaxis is associated with long term benefit for the baby.

\section{Appendix}

\section{Sample size calculations}

Original sample size calculation based on data in the literature on the "fFN +" group

A sample size of 750 (375 per group) gives adequate statistical power to detect clinically important and plausible differences in the three primary measures of outcome. All these power calculations allow for loss to follow up rates and compliance. Delivery $<34$ weeks on placebo is expected to be $40 \%$ (data from an untreated high risk UK population with a positive fFN test at 22 weeks [27] and $27 \%$ on progesterone consistent with the odds ratio of 0.45 for the overall PTB with any progestational agent in the most recent systematic review [28]. With 750 randomised, the study will have 95\% power at a $5 \%$ level of significance to detect such a reduction from $40 \%$ to $27 \%$ using a two-sided binomial test. For a more modest reduction from $40 \%$ to $30 \%$ (odds ratio 0.64 ) the study would still have $80 \%$ power. For the neonatal outcome, the Meis study found 7.2\% died, $14.6 \%$ had CLD and $5.2 \%$ had IVH on placebo, compared to $4.6 \%$ death, $8.6 \%$ CLD and $1.3 \%$ IVH on progesterone [4]. Our neonatal primary outcome is a composite of these and also includes non-haemorrhagic brain injuries. With $\mathrm{n}=750$ randomised, the
OPPTIMUM study would have $80 \%$ power at a $5 \%$ level of significance to detect a difference in this composite outcome of death, brain damage, or chronic lung disease from 20 to $12 \%$, using a binomial test.

The childhood developmental outcome at 2 years will be assessed using the Bayley III Cognitive Scale which correlates well with later IQ. With 750 randomised, the study will have $93 \%$ power at a $5 \%$ level of significance to detect a difference in means equivalent to 0.25 of a standard deviations, using a two sample two sided $t$-test. Based on previous work [29], we estimate the standard deviation will be about 15 points, enabling us to detect a difference of 4 points in the Bayley Score. In clinical terms, a difference of 4 points is small, thus the power of the study to detect larger, more clinically significant differences, is high. We have powered this outcome at over $90 \%$ to make some allowance for a possible dilution of the power due to incorporating the deaths (using a two stage statistical model).

The revised sample size calculation below, based on blinded data (as of April 2011) and inclusion of the "fFN"- group is around 1250 participants

Obstetric outcome (delivery $<34$ weeks).

The table below gives the estimated power for three combinations of sample size (all 1:2 fFN+/fFN- groups, assuming 375:750 (total 1125), 400:800 (total 1200) and 425:850 (total 1275) assuming (fFN+, fFN-) event rates (delivery $<34$ weeks) of $(40 \%, 10 \%),(45 \%, 13 \%)$, and $(45 \%, 15 \%)$. All calculations assume a relative treatment effect of $32.5 \%$.

The justification for these combinations is as follows:

1. The $40 \%$ untreated rate is as per the original assumption. We have looked at the blinded (aggregated) event rate for the first 67 women randomised in OPPTIMUM (ignoring any possible bias created by recording the early births before later births in the most recently randomised). For the fFN + group, 29/67 (43\%, 95\% exact confidence interval $31 \%$ to $56 \%$ ) had delivered at $<34$ weeks. The blinded event rate according to the assumed $40 \%$ untreated rate with a $27 \%$ treated rate $(32.5 \%$ relative reduction) would be $33.5 \%$. Although the numbers are relatively small, there appears to be a higher event rate than anticipated, which would be consistent with having selected a higher risk and hence scarcer subset than we planned for). An overall blinded rate of $43 \%$ could comprise an untreated rate of $52 \%$ and a treated rate of $35 \%$. We assume conservatively that the baseline event rate in the untreated fFN + group is $45 \%$. 
2. For the fFN- group women data on the first 24 women suggested that $8 / 24$ (33\%, exact $95 \%$ confidence interval $16 \%$ to $55 \%$ ) had delivered early. As indicated above, this percentage is likely to be inflated since we record the early deliveries before observing the term deliveries. However, fortunately, we also had followed up those who were not randomised (by definition, all of whom had a negative fFN test) into OPPTIMUM from the start. Of the 398 followed, 32 have had a delivery $<34$ weeks. We know that $57 \%$ of these 398 would have been eligible for the trial (short cervix $<25 \mathrm{~mm}$ ), and the epidemiology indicates that it is very unlikely to give birth $<34$ weeks with none of the classic risk factors. So we conservatively estimate that around $13 \%$ (i.e. around $29 / 225$, exact confidence interval $9 \%$ to $18 \%$ ) of those recruited into the fFN- group, based on OPPTIMUM data, will have a delivery $<34$ weeks.

The conclusion is that it seems likely that if we recruit at least 1125 (375:750) we will have almost $90 \%$ for the primary obstetric outcome, assuming a slightly higher rate in the fFN + group (45\% rather than $40 \%$ ) and a corresponding untreated event rate of $13 \%$ in the fFNwomen. If as we expect from the early returns the fFNevent rate is $>15 \%$, we will be comfortably powered at the original $95 \%$ for the obstetric outcome. Table 1 shows the various power scenarios.

The neonatal outcome (neonatal death, severe chronic lung disease, intraventricular haemorrhage).

Data from the first cohort of women randomised (and in particular those randomised in the low risk group) into OPPTIMUM was even sparser for this outcome, as it is determined by convention to up to 30 days after a full term birth. For all those randomised with mature data at the time of analysis, 11/68 have had the composite neonatal outcome $(16 \%$, exact $95 \%$ confidence interval of $8 \%$ to $27 \%$ ). Given that the rate of pre-term birth is higher than anticipated, it seems reasonable to inflate the assumed neonatal event rate from 20\% to $25 \%$ (giving an blinded event rate of $20 \%$, assuming a $40 \%$ relative reduction - and $20 \%$ is very consistent with the observed $95 \%$ confidence interval of $8 \%$ to $27 \%$ ). If we then further assume a pro-rata event rate in line with the rates of delivery $<34$ weeks on the obstetric outcome, with the fFN- having roughly one-third the events of the fFN + i.e. $8 \%$ rather than $25 \%$, then Table 2 below shows the various power scenarios:

We conclude that by randomising at least $375 \mathrm{fFN}+$ and $750 \mathrm{fFN}$ - women, assuming an untreated rate of $25 \%$ of the composite of neonatal death, severe chronic lung disease, intraventricular haemorrhage in the fFN+, and $8 \%$ in the $\mathrm{fFN}$-, with an assumed $40 \%$ relative
Table 1 Power scenarios for the neonatal outcome

\begin{tabular}{cccc}
\hline $\mathbf{f F N}+$ & fFN- & Subjects (fFN+/fFN-) [Total] & Power \\
\hline $40 \%$ & $10 \%$ & $375 / 750(1125)$ & $81 \%$ \\
& & $400 / 800(1200)$ & $83 \%$ \\
& & $425 / 850(1275)$ & $85 \%$ \\
$45 \%$ & $13 \%$ & $375 / 750(1125)$ & $88 \%$ \\
& & $400 / 800(1200)$ & $90 \%$ \\
& & $425 / 850(1275)$ & $92 \%$ \\
$50 \%$ & $15 \%$ & $375 / 750(1125)$ & $93 \%$ \\
& & $400 / 800(1200)$ & $94 \%$ \\
& & $425 / 850(1275)$ & $95 \%$ \\
\hline
\end{tabular}

treatment effect (as per original calculation) the original power of the study for this neonatal outcome will be preserved at $>80 \%$ power.

The childhood development outcome (Bayley Score at 2 years).

There are no data yet on this outcome within OPPTIMUM. In the original calculation we assumed that the difference in mean Bayley score would be 4 units with a common standard deviation of 15 units. It is more difficult to assess the power convincingly with a mixture of fFN + and fFN- women on a continuous outcome such as the Bayley Score, since the power calculation requires assumptions about not just the anticipated treatment effect but also the assumed variability via the standard deviation. If we assume the same 4 unit difference in the fFN + and a $4 / 3$ unit difference in the fFN- group (consistent with the pro-rata rate of delivery $<34$ weeks), with the same 15 unit standard deviation, then the study will have $71 \%, 73 \%$, and $76 \%$ power if 1125,1200 , or 1275 are randomised (375:750, 400:800; and 425:850) as per Tables 1 and 2 above. However, this is for an unadjusted analysis, and in practice we will adjust for fFN + and fFN- group, and a limited number of other baseline covariates strongly related to Bayley Score at 2 years (e.g. gender) as specified in the statistical analysis plan, and this will reduce the variability and hence increase the power. For example, if the underlying variability in the lower risk group is lower - say halved, at 7.5 units, consistent with a higher proportion having uniformly high Bayley Scores since they have no disability - then the approximate power would be 93\%, 94\%, and $95 \%$ respectively for 1125,1200 , or 1275

Table 2 Power scenarios for the obstetric outcome

\begin{tabular}{lccc}
\hline $\mathbf{f F N}+$ & fFN- & Subjects (fFN+/ fFN-) [Total] & Power \\
\hline $25 \%$ & $8 \%$ & $375 / 750(1125)$ & $81 \%$ \\
& & $400 / 800(1200)$ & $83 \%$ \\
& & $425 / 850(1275)$ & $86 \%$ \\
\hline
\end{tabular}


randomised in total. In practice the reduction in variability by adjusting for both this design variate ( $\mathrm{fFN}+$ and fFN- ) and additional baseline covariates may be considerably greater, so we are confident that the original power on the childhood development outcome will be protected at or above the original $90 \%$ level by randomising at least 1125 subjects (375:750).

\section{Endnotes}

ahttp://www.fda.gov/NewsEvents/Newsroom/

PressAnnouncements/ucm242234.htm

bhttp://www.cbsnews.com/8301-505245_162-57363066/

fda-panel-votes-down-preterm-birth-gel/

chttp://www.rcog.org.uk/womens-health/guidelines/ use-progesterone-prevent-preterm-delivery

\section{Competing interests}

JEN has received research grants from (non commercial) funding agencies for pregnancy related conditions - full list available on request, was a paid consultant to a small drug company (Preglem) with an interest in obstetric /gynaecological drugs - <£5,000 2010 - 2012, and an unpaid consultant to Hologic (who manufacture fibronectin amongst others).

ST has received research grants from commercial and non-commercial funding agencies for pregnancy-related research. Full list available on request. ST is paid or unpaid consultant for GSK, Ferring and Hologic. PB has acted as a consultant for GSK.

AS receives funds for educational purposes from Hologic, Ferring, GSK and Alere. He sits on advisory boards for Roche, GSK and Hologic. AS receieves research funds from Alere and Hologic which are paid to his institution.

\section{Author' contributions}

The protocol was jointly devised by all the authors with the exception of SW. JEN prepared the first draft of this manuscript for submission. All authors commented and agreed the final draft.

\section{Disclaimer}

This report is independent research funded by the MRC and managed by the NIHR on behalf of the MRC-NIHR partnership. The views expressed in this publication are those of the author(s) and not necessarily those of the MRC, NHS, NIHR or the Department of Health.

\section{Author details \\ ${ }^{1}$ University of Edinburgh MRC Centre for Reproductive Health, The Queen's Medical Research Institute, 47 Little France Crescent, Edinburgh EH16 4TY, UK. 'Women's Health Academic Centre, King's Health Partners, 10th floor North Wing, St.Thomas' Hospital, London SE1 7EH, UK. ${ }^{3}$ Imperial College Faculty of Medicine, Institute for Reproductive and Developmental Biology, Hammersmith Hospital Campus, Du Cane Road, London W12 0NN, UK. ${ }^{4}$ Peninsula College of Medicine and Dentistry, Peninsula Medical School, Barrack Road, Exeter EX2 5DW, UK. Institute of Cellular Medicine, Uterine Cell Signalling Group, 3rd Floor, William Leech Building, The Medical School, Newcastle University, Newcastle NE2 4HH, UK. ${ }^{6}$ Institute for Women's Health, University College London, Room 244, Medical School Building, 74 Huntley Street, London WC1E 6AU, UK. ${ }^{7}$ Centre for Healthcare Randomised Trials (CHaRT), Health Services Research Unit, 3rd Floor Health Sciences Building, University of Aberdeen, Foresterhill, Aberdeen AB25 2ZD, UK. ${ }^{8}$ University of Warwick Division of Health Sciences, Warwick Medical School, The University of Warwick, Coventry CV4 7AL, UK. ' Histopathology Department, Department of Paediatric Laboratory Medicine, Level 3, Camelia Botnar Laboratories, Great Ormond Street Hospital, London WC1N 3JH, UK. ${ }^{10}$ School of Nursing, Midwifery and Social Work, The University of Manchester, Manchester, UK. ${ }^{11}$ University of Edinburgh MRC Centre for Reproductive Health, c/o Simpson Centre for Reproductive Health, Royal Infirmary, 51 Little France Crescent, Edinburgh EH16 4SA, UK.}

Received: 30 May 2012 Accepted: 28 June 2012

Published: 6 August 2012

\section{References}

1. March of Dimes Foundation: March of Dimes White paper on preterm birth: the global and regional toll. White Plains, New York: March of Dimes foundation; 2009.

2. Norman JE, Morris C, Chalmers J: The effect of changing patterns of obstetric care in Scotland (1980-2004) on rates of preterm birth and its neonatal consequences: perinatal database study. PLoS Med 2009, 6(9):e1000153.

3. Goldenberg RL, Culhane JF, lams JD, Romero R: Epidemiology and causes of preterm birth. Lancet 2008, 371(9606):75-84.

4. Arbyn M, Kyrgiou M, Simoens C, Raifu AO, Koliopoulos G, Martin-Hirsch P, Prendiville W, Paraskevaidis E: Perinatal mortality and other severe adverse pregnancy outcomes associated with treatment of cervical intraepithelial neoplasia: meta-analysis. BMJ (Clinical Res Ed) 2008, 337:a1284.

5. da Fonseca EB, Bittar RE, Carvalho MH, Zugaib M: Prophylactic administration of progesterone by vaginal suppository to reduce the incidence of spontaneous preterm birth in women at increased risk: a randomized placebo-controlled double-blind study. Am J ObstetGynecol 2003, 188(2):419-424.

6. Fonseca EB, Celik E, Parra M, Singh M, Nicolaides KH, Thornton S, Alfirevic Z, Smith G, Radhakrishnan P, Khoury O, et al: Progesterone and the risk of preterm birth among women with a short cervix. N Engl J Med 2007, 357(5):462-469.

7. Meis PJ, Klebanoff M, Thom E, Dombrowski MP, Sibai B, Moawad AH, Spong CY, Hauth JC, Miodovnik M, Varner MW, et al: Prevention of recurrent preterm delivery by 17 alpha-hydroxyprogesterone caproate. $N$ Engl J Med 2003, 348(24):2379-2385.

8. Hassan SS, Romero R, Vidyadhari D, Fusey S, Baxter J, Khandelwal M, Vijayaraghavan J, Trivedi Y, Soma-Pillay P, Sambarey P, et al: Vaginal progesterone reduces the rate of preterm birth in women with a sonographic short cervix: a multicenter, randomized, double-blind, placebo-controlled trial. Ultrasound Obstet Gynecol 2011, 38:18-31.

9. Dodd JM, Flenady V, Cincotta R, Crowther CA: Prenatal administration of progesterone for preventing preterm birth. Cochrane Database Syst Rev 2006, 1:CD004947.

10. Dodd JM, Flenady VJ, Cincotta R, Crowther CA: Progesterone for the prevention of preterm birth: a systematic review. Obstet Gynecol 2008, 112(1):127-134.

11. Romero R, Nicolaides K, Conde-Agudelo A, Tabor A, O'Brien JM, Cetingoz E, Da Fonseca E, Creasy GW, Klein K, Rode L, et al: Vaginal progesterone in women with an asymptomatic sonographic short cervix in the midtrimester decreases preterm delivery and neonatal morbidity: a systematic review and metaanalysis of individual patient data. Am J Obstet Gynecol 2012, 206(2):124 e121-119.

12. Northen AT, Norman GS, Anderson K, Moseley L, Divito M, Cotroneo M, Swain M, Bousleiman S, Johnson F, Dorman K, et al: Follow-up of children exposed in utero to 17 alpha-hydroxyprogesteronecaproate compared with placebo. ObstetGynecol 2007, 110(4):865-872.

13. Progesterone and preterm birth prevention: translating clinical trials data into clinical practice. Am J Obstet Gynecol 2012, 206(5):376-386.

14. Junpeng M, Huang $S$, Qin S: Progesterone for acute traumatic brain injury. Cochrane Database Syst Rev 2011, 1:CD008409.

15. Kenyon SL, Taylor DJ, Tarnow-Mordi W: Broad-spectrum antibiotics for spontaneous preterm labour: the ORACLE II randomised trial. ORACLE Collaborative Group. Lancet 2001, 357(9261):989-994.

16. Kenyon S, Pike K, Jones DR, Brocklehurst P, Marlow N, Salt A, Taylor DJ: Childhood outcomes after prescription of antibiotics to pregnant women with spontaneous preterm labour: 7-year follow-up of the ORACLE II trial. Lancet 2008, 372(9646):1319-1327.

17. Hoover RN, Hyer M, Pfeiffer RM, Adam E, Bond B, Cheville AL, Colton T, Hartge $P$, Hatch EE, Herbst AL, et al: Adverse health outcomes in women exposed in utero to diethylstilbestrol. N Engl J Med 2011, 365(14):1304-1314.

18. Anon: Disability and Perinatal care: report of two working groups. Oxford: NEPU and Oxford HA; 1995.

19. Modi N, Dore CJ, Saraswatula A, Richards M, Bamford KB, Coello R, Holmes A: A case definition for national and international neonatal bloodstream infection surveillance. Arch Dis Child 2009, 94(1):F8-F12. 
20. Standards for hospitals providing neonatal intensive and high dependency care and categories of babies requiring neonatal care. 2 nd edition.: British Association of Perinatal Medicine; 2001. http://www.bapm.org/publications/ documents/guidelines/hosp_standards.pdf.

21. BAPM/RCPCH Working Group Report: Classification of health status at 2 years as a perinatal outcome. 2008

22. Edwards IR, Biriell C: Harmonisation in pharmacovigilance. Drug Saf 1994, 10(2):93-102.

23. Honest H, Forbes CA, Duree KH, Norman G, Duffy SB, Tsourapas A, Roberts $T E$, Barton PM, Jowett SM, Hyde CJ, et al: Screening to prevent spontaneous preterm birth: systematic reviews of accuracy and effectiveness literature with economic modelling. Health Technol Assess 2009, 13(43):1-627.

24. Lachenbruch PA: Analysis of data with excess zeros. Stat Methods Med Res 2002, 11(4):297-302

25. Farine D, Mundle WR, Dodd J, Basso M, Delisle MF, Grabowska K, Hudon L, Menticoglou SM, Murphy-Kaulbeck LC, Ouellet A, et al: The use of progesterone for prevention of preterm birth. J Obstet Gynaecol Can 2008, 30(1):67-77.

26. American College of Obstetricians and Gynecologists: ACOG committee opinion no 419.on "The use of progesterone to prevent preterm birth". American College of Obstetricians and Gynecologists. Obstet Gynecol 2008, 112:963-965.

27. Shennan A, Jones G, Hawken J, Crawshaw S, Judah J, Senior V, Marteau T, Chinn S, Poston L: Fetal fibronectin test predicts delivery before 30 weeks of gestation in high risk women, but increases anxiety. BJOG 2005, 112(3):293-298.

28. Sanchez-Ramos L, Kaunitz AM, Delke I: Progestational agents to prevent preterm birth: a meta-analysis of randomized controlled trials. Obstet Gynecol 2005, 105(2):273-279.

29. Wolke D, Meyer R: Ergebnisse der Bayerischen Entwicklungstudie: implikationen fur theorie und praxis. Kindheit und Entwicklung 1999, 8:24-36.

doi:10.1186/1471-2393-12-79

Cite this article as: Norman et al:: Trial protocol OPPTIMUM- Does progesterone prophylaxis for the prevention of preterm labour improve outcome?. BMC Pregnancy and Childbirth 2012 12:79.

\section{Submit your next manuscript to BioMed Central and take full advantage of:}

- Convenient online submission

- Thorough peer review

- No space constraints or color figure charges

- Immediate publication on acceptance

- Inclusion in PubMed, CAS, Scopus and Google Scholar

- Research which is freely available for redistribution 